\title{
Robot-assisted mandibular angle osteotomy using electromagnetic navigation
}

\author{
Mengzhe Sun ${ }^{1 \#}$, Li Lin ${ }^{1,2 \#}$, Xiaojun Chen ${ }^{1}$, Cheng Xu' ${ }^{2}$, Mar Aung Zin ${ }^{1}$, Wenqing Han ${ }^{1}$, Gang Chai ${ }^{1,3,4}$ \\ ${ }^{1}$ Department of Plastic and Reconstructive Surgery, Shanghai 9th People's Hospital, School of Medicine, Shanghai Jiao Tong University, Shanghai, \\ China; ${ }^{2}$ Institute of Forming Technology \& Equipment, Shanghai Jiao Tong University, Shanghai, China; ${ }^{3}$ School of Medical Instrumentation, \\ Shanghai University of Medicine \& Health Sciences, Shanghai, China; ${ }^{4}$ Department of Plastic and Reconstructive Surgery, Maternal and Child \\ Health Care Hospital of Hainan Province, Haikou, China \\ Contributions: (I) Conception and design: M Sun; (II) Administrative support: G Chai; (III) Provision of study materials or patients: L Lin, C Xu, X \\ Chen, MA Zin; (IV) Collection and assembly of data: W Han; (V) Data analysis and interpretation: W Han, G Chai; (VI) Manuscript writing: All \\ authors; (VII) Final approval of manuscript: All authors. \\ \#These authors contributed equally to this work as co-first authors. \\ Correspondence to: Professor Gang Chai, MD, PhD. Department of Plastic and Reconstructive Surgery, Shanghai 9th People's Hospital, School of \\ Medicine, Shanghai Jiao Tong University, 639 Zhi Zao Ju Rd, Shanghai 200011, China. Email: chaig1081@sh9hospital.org.cn.
}

Background: To explore the potential of electromagnetic (EM) navigation technology in the field of robotassisted surgery, we set up a maxillofacial surgical robotic system (MSRS) guided by an EM navigation tool. Mandibular angle osteotomy was used to analyze the feasibility in confined surgical areas.

Methods: Model and animal experiments were implemented to validate the system precision. Before the experiment, a customized dental splint was made and then fixed with a standard navigation part. An accurate 3D surgical plan was designed based on the preoperative CT scan. During the experiment, the splint was rigidly mounted on teeth for navigation registration, so the robot could position a specially designed template to guide the accurate osteotomy according to the preoperative plan. For the model experiment, a Coordinate Measuring Machine was used to measure the template's position and angle. For the animal experiment, surgeons completed the surgery by moving a saw along the template, while a postoperative CT scan was carried out to calculate the precision.

Results: All procedures were successfully completed, with no complications in any of the experimental animals. In the model experiment, the accuracy of the navigation position and angle was $0.44 \pm 0.19 \mathrm{~mm}$ and $3.5^{\circ} \pm 2.1^{\circ}$, respectively. In the animal experiment, the lateral osteotomy line error was $0.83 \pm 0.62 \mathrm{~mm}$, the interior error was $1.06 \pm 1.03 \mathrm{~mm}$, and the angle between the actual cutting plane and preoperative planning plane was $5.9^{\circ} \pm 4.7^{\circ}$.

Conclusions: Robot-assisted surgery with EM navigation resulted feasible in the real operating environment. Moreover, this system's precision could meet clinical needs, while the proposed procedure was safe and easy on animals. Consequently, this approach has the potential to be applied to clinical craniomaxillofacial practice in the near future.

Keywords: Robot-assisted surgery (RAS); electromagnetic (EM) navigation; cranio-maxillofacial surgery

Submitted Sep 07, 2020. Accepted for publication Jan 08, 2021.

doi: 10.21037/atm-20-6305

View this article at: http://dx.doi.org/10.21037/atm-20-6305 


\section{Introduction}

According to the oriental aesthetic standards, a square face is viewed as coarse and masculine (1). Mandibular angle osteotomy is a common cosmetic surgery in Asia used to improve facial contour. In such type of aesthetic surgery dealing with the face, surgical accuracy and symmetry are very important in postoperative evaluation. The incision is made intraorally, so inevitably, the operation space is limited, and the surgeon has a poor operation field. Complications such as vascular injury, nerve damage, facial paralysis, mandible fracture, and bilateral asymmetry can easily occur due to the confined intraoperative space. A preoperative plan is made to avoid these situations; however, surgeons may have trouble determining the osteotomy line and performing the surgery as planned as they cannot see the mandible clearly and completely.

Surgical navigation technology, which is an effective solution, was first introduced in neurosurgery in 1908 by Horsley and Clarke, who put the intraoperative application of surgical planning into practice (2). Electromagnetic (EM) navigation is an upcoming tool in craniomaxillofacial surgery (3). The magnetic field force is used as a noncontact cross-space action mode, and the use of a magnetic field for establishing location is flexible and convenient and does not require bulky equipment. It is also not limited by blocking issues. Field sensors fixed on the targets pick up its real-time position information in the magnetic field so as to achieve the tracking and navigation of the surgical object and instrument. The point of surgical navigation is to carry out spatial registration of preoperative plan, actual field, and surgical instruments. Precise registration is crucial because it can directly affect the precision of all subsequent navigation tasks (4). Robotic aids are naturally suited for this alignment and stabilization task, thus reducing the surgeon's manual error and potentially minimizing complication rates (5). Currently, there is no well-developed system in craniomaxillofacial surgery that combines EM navigation with robot assistance. Therefore, we set up a maxillofacial surgical robotic system (MSRS) guided by an EM navigation tool so as to help the surgeons accurately perform surgery according to the preoperative plan.

After establishing the system and workflow on the mandible model in the laboratory, animal experiments were conducted in a real operating environment, implementing post-operation CT to evaluate the accuracy of the system. Beagles were chosen because of their stable strain and strong vitality. By comparing outcomes with traditional methods, we intended to verify the feasibility of this procedure.

We present the following article in accordance with the ARRIVE reporting checklist (available at http://dx.doi. org/10.21037/atm-20-6305).

\section{Methods}

\section{System}

Our MSRS consisted of two major parts: the robot and the navigation equipment. The former included an industrial robotic arm UR5 (Universal Robots) with a self-designed template clamped on the end, a base station equipped with a computer for surgical planning and robotic control. The latter consisted of the Aurora V3 (Northern Digital Inc.) EM tracking system, which included a system control unit, a field generator, and sensors, as well as the navigation splint assembled by a customized dental splint and a standard navigation part.

Model and animal experiments were implemented to validate the precision of the system (Figure 1). Animal experimental workflow was provided in supplementary files (Video 1). Model experiment with 5 mandible models was carried out in the following order: navigation splint making, preoperative CT, surgical planning, navigation registration, template positioning, and measuring. Animal experiment was carried out in two groups with 4 mandibular angle osteotomies (experimental unit) each. The experimental group underwent: navigation splint making, preoperative CT, surgical planning, navigation registration, template positioning, osteotomy, postoperative CT, postoperative measurements. The control group: preoperative CT, surgical planning, traditional osteotomy, postoperative CT, postoperative measurements.

\section{Subjects}

Five mandible models were 3d-printed (ProJet 660, 3D system, USA) based on patient data from the digital laboratory in Shanghai 9th People's Hospital (Philips, Amsterdam, The Netherlands).

Four 1-year-old test naïve beagles, 2 males, and 2 females, weighing 8 to $10 \mathrm{~kg}$, were selected as animal experiment subjects, and randomly divided into two groups. All animals were provided by the hospital animal house with an animal use permit (NO. SYXK2015-0002, General grade). The room temperature was maintained in the range 


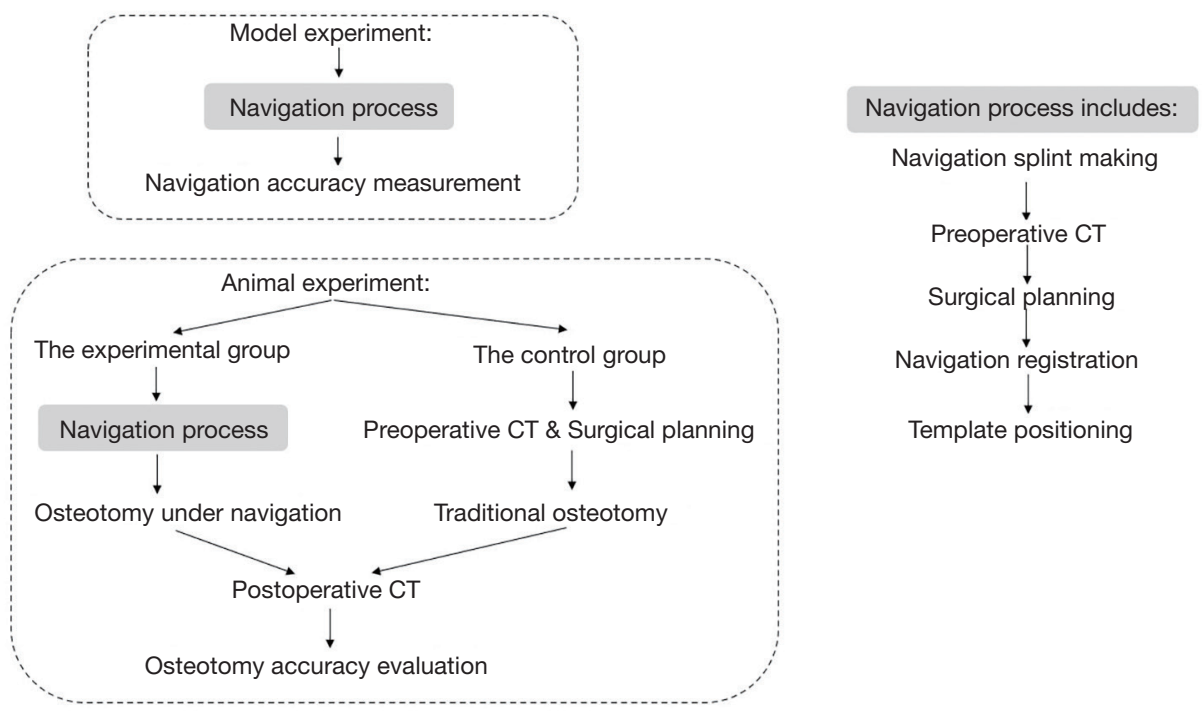

Figure 1 Overall experiment workflow diagram.

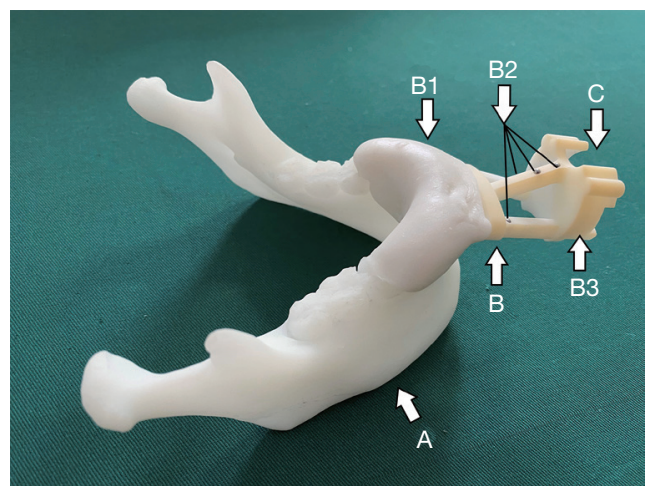

Figure 2 Navigation splint mounted on teeth of the mandible model. (A: mandible model; B: navigation splint; B1: customized dental splint; B2: steel spheres; B3: navigation standard part; C: dent to install EM sensor).

of $22-23{ }^{\circ} \mathrm{C}$, and the relative humidity was in the range of $40-45 \%$.

\section{Ethical statement}

Experiments were performed under a project license (No.: SH9HIEC-2017-319-T239) granted by Independent Ethics Committee of Shanghai Ninth People's Hospital affiliated with the Shanghai Jiao Tong University, School of Medicine, in compliance with institutional relevant guidelines and regulations for the care and use of animals.

\section{Navigation splint making}

A customized dental splint was made based on the patient's dental mold, then assembled with a standard navigation part, which was fixed with four steel spheres with a diameter of $2 \mathrm{~mm}$ as the magnetic field tracking targets for navigation (Figure 2). Preoperative CT scans (Philips Brilliance 64 CT scanner, $284 \mathrm{~mA}, 120 \mathrm{kV}$, matrix $512 \times 512,0.625 \mathrm{~mm}$ layer $3 \mathrm{D}$ reconstruction) were carried out with the splint mounted on teeth.

\section{Surgical planning}

In the three-dimensional reconstruction software (Mimics19.0, Materialise, Belgium), the patient's DICOM data was imported, and by segmenting the proper threshold value of CT, the mandible was reconstructed, and the osteotomy line was designed so as to avoid the inferior alveolar nerve running inside the mandible (Figure 3). The mandibular angle osteotomy surgical plan was saved in STL form and then imported to the robot with relative location information of four steel spheres.

\section{Registration and navigation}

After fixation of the mandible model and magnetic generator, the splint was mounted on the mandible model's teeth to reflect the position of the mandible. Magnetic field receptors were connected to the robot and the navigation 


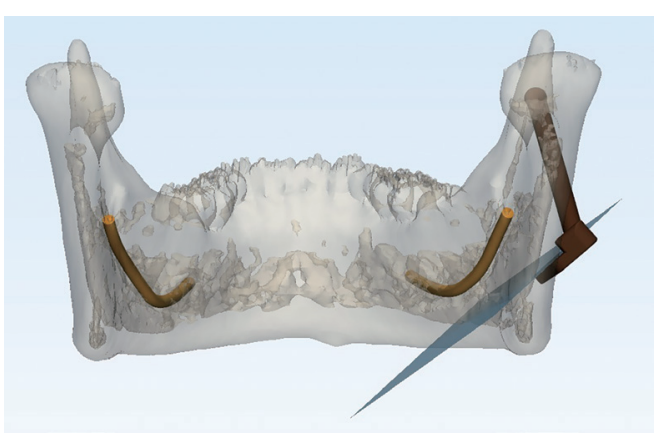

Figure 3 Surgical plan of mandibular angle osteotomy. (Grey: mandible; yellow: inferior alveolar nerve; blue: osteotomy plane; brown: template).

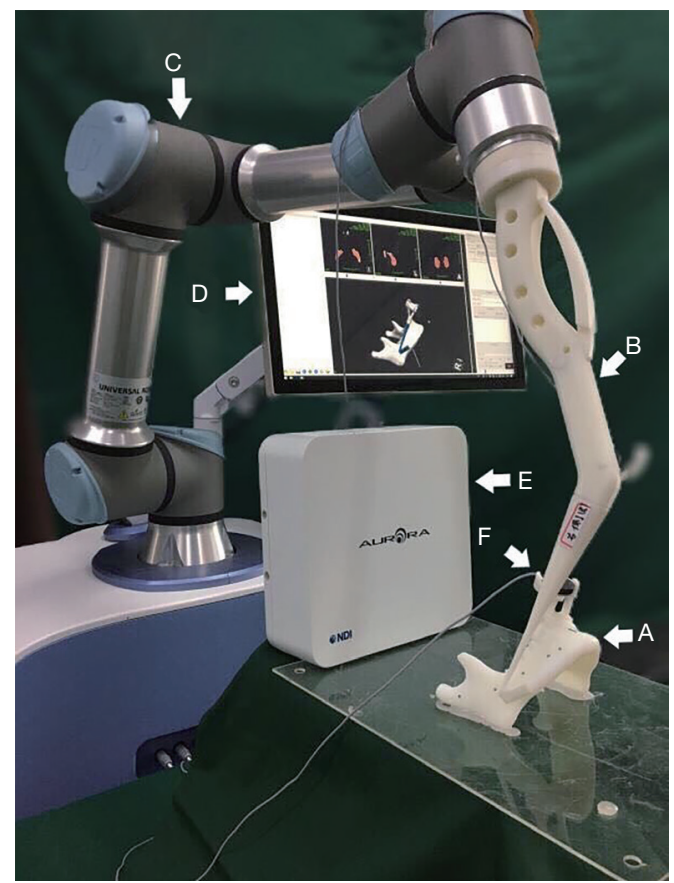

Figure 4 Model experiment of our maxillofacial surgical robotic system (MSRS). (A: mandible model mounted with navigation splint; B: template; C: robot; D: computer; E: EM generator; F: EM sensor).

splint for tracking the robot and mandible movement intraoperatively via the magnetic navigation system. The registration between virtual data and the actual space was automatically completed by the software via matrix transformation using four steel spheres as registration points. The positions of the robot, the mandible model, and the osteotomy surgical plan were all aligned after

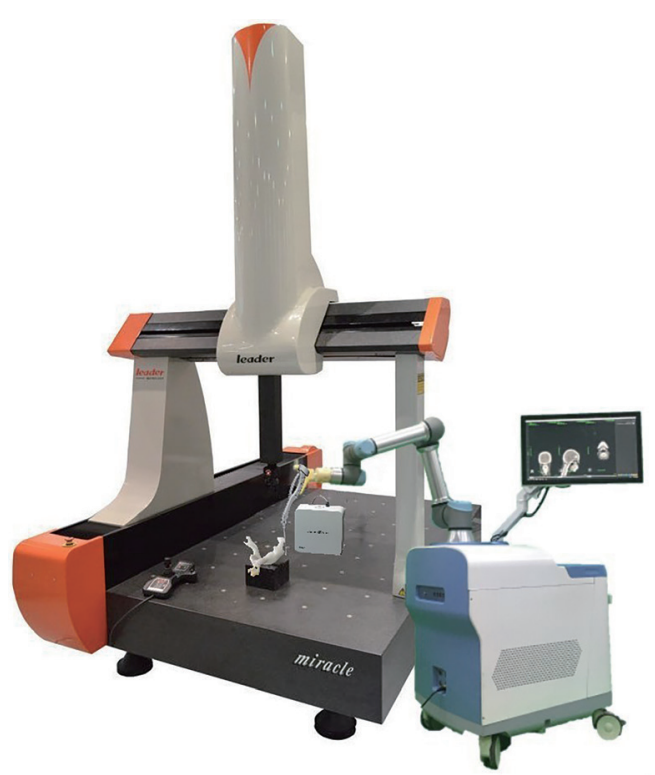

Figure 5 Schematic diagram of navigation accuracy measurement.

registration. It was ensured that the robot base and operation table made no movement after confirming the relative position between the robot and the mandible in the magnetic field. The registration process of our MSRS can be visualized in the navigation software interface.

To guide the position and angle of the osteotomy intraoperatively, we designed and $3 \mathrm{~d}$-printed a template, which was clamped on the end of the robot. The robot moved the template to the target location according to the pre-imported surgical plan (Figure 4).

\section{Navigation accuracy measurement}

Coordinate Measuring Machine (Miracle NCT685) was used to measure the accuracy of navigation positioning (Figure 5). By tapping the probe stylus on the edge and osteotomy plane of the template, we acquired the navigation position and angle values. The same operation was performed on registration points of the mandible model, and the expected position and angle of osteotomy could be obtained by matrix conversion. Then, the actual three-dimensional coordinate value was compared with the expected value to calculate the magnetic navigation accuracy.

\section{Animal experiment}

In order to verify the feasibility of this system in a real operation environment, animal experiments were conducted 


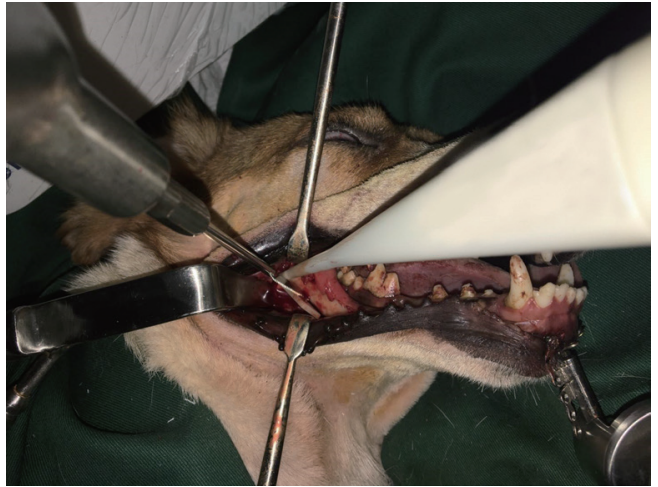

Figure 6 Robot-assisted mandibular angle osteotomy by moving the saw along the navigated template.

(Video 1). The sample size was calculated using the formula $n=\left[\frac{Z_{\alpha / 2}+Z_{\beta}}{\delta / \sigma}\right]^{2}\left(Q_{1}^{-1}+Q_{2}^{-1}\right)$. If $\mathrm{n}_{1}=\mathrm{n}_{2}$, then $\mathrm{Q}_{1}=\mathrm{Q}_{2}=0.5$. Population standard deviation $(\mathrm{mm})$ was from the previous model experiment, and the difference between two groups $\delta(\mathrm{mm})$ was set empirically. To achieve power $(1-\beta)=0.9$ and $\alpha=0.05, n=\left[\frac{1.96+1.282}{0.5 / 0.19}\right]^{2}\left(0.5^{-1}+0.5^{-1}\right) \approx 6.1, \mathrm{n}_{1}$ and $\mathrm{n}_{2}$ should be no less than 3 . In case of an accident, we prepared one more case for each group.

Four robot-assisted surgeries and four traditional manual surgeries were alternately performed by the same surgical team between August and December 2019. The surgeon performing the osteotomy was an attending who had been practicing plastic surgery for ten years. Before surgery, all animals underwent fasting for 12 hours, and then received an intramuscular injection of $0.6-0.8 \mathrm{~mL}$ of Lumianning (Xylazine $20 \mathrm{mg} / \mathrm{mL}$ ) to calm them down and to ease the follow-up intravenous injection. For anesthesia, 3\% Pentobarbital with a dose of $1 \mathrm{~mL} / \mathrm{kg}$ was administered intravenously without intraoperative intubation. All these were done by the animal laboratory technician, so experimenters did not know the animal's number until the operation began and the blinding of the surgeons could be achieved.

After anesthesia, the dog's mandible was dissected and exposed intraorally. Then the mandible was fixed to the operation table using a bedside fixator (BERCHTOLD OZ1251900) and the splint was mounted on the teeth. The next steps were the same as the model experiment.

After navigation, the osteotomy operation was carried out by the surgeon moving the saw along the template (Figure 6). During the osteotomy, EM system equipment could be removed to make more room as long as the mandible did not move. Each of the four beagles had both mandible angles removed, while only half was robot-assisted and the other half was done traditionally (surgeon looked at the preoperative plan to figure out an approximate position on the mandible and made the osteotomy empirically).

\section{Osteotomy accuracy evaluation}

The animals were kept in single cages and were allowed free access to food and water for two weeks postoperatively to observe the corresponding complications. Postoperative CT was used to measure the robot-assisted surgery accuracy. Imaging DICOM data was imported to Mimics software to reconstruct the mandible and later imported in STL form to Geomagic Control (3D Systems) along with the preoperative plan for accuracy evaluation (Figures 7,8). This step was done by another researcher to prevent bias. Osteotomy accuracy was used as the primary outcome: 10 points were measured along both lateral and interior osteotomy line. In addition, three secondary outcomes were evaluated: operation time, osteotomy angle deviation, and postoperative complications.

\section{Statistical analysis}

An independent sample $t$-test was performed to compare the differences between the two groups. Each group of animals was an experimental unit. If $\mathrm{P}<0.05$, the data were considered as meeting the assumption that robot-assisted surgery had better outcomes than the traditional control group.

\section{Results}

The model experiment was carried out successfully in all 5 mandible models (Table 1). Equipment installation and navigation process took 15 minutes on average. The mean error of osteotomy position and angle compared with preoperative plan was $0.44 \pm 0.19 \mathrm{~mm}$ and $3.5^{\circ} \pm 2.1^{\circ}$, respectively.

In the animal experiment, all beagles were healthy and test naive before surgery. The result of the experiment is shown in Table 2. According to postoperative CT: analysis of all osteotomies (4/4) in robot-assisted group revealed that the average error along lateral osteotomy line was $0.83 \pm 0.62 \mathrm{~mm}$, interior line error was $1.06 \pm 1.03 \mathrm{~mm}$, and the angle between actual cutting plane and preoperative planning plane was $5.9^{\circ} \pm 4.7^{\circ}$; As for osteotomies (4/4) 


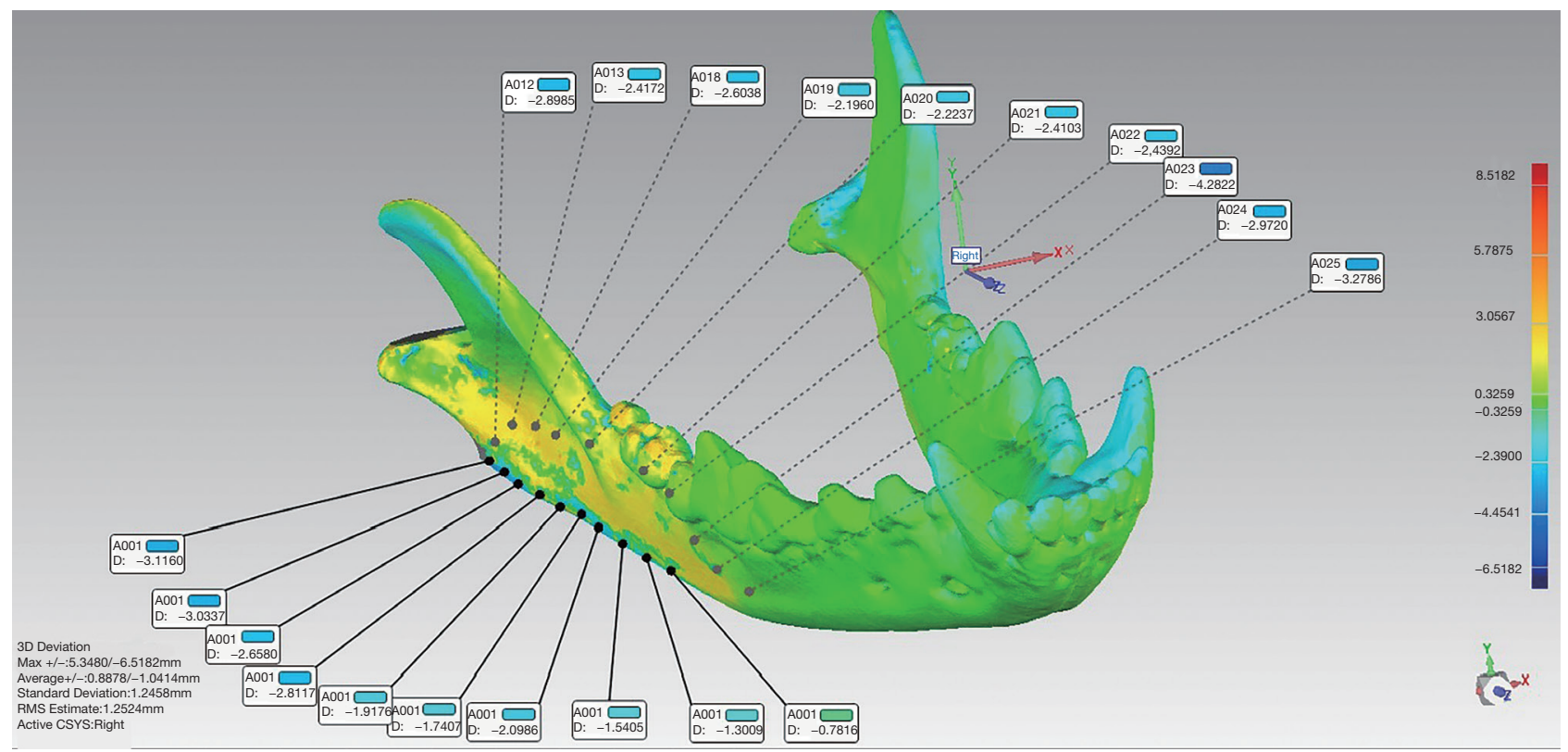

Figure 7 Ten points were measured along both the lateral and interior osteotomy line to calculate the deviation of position.

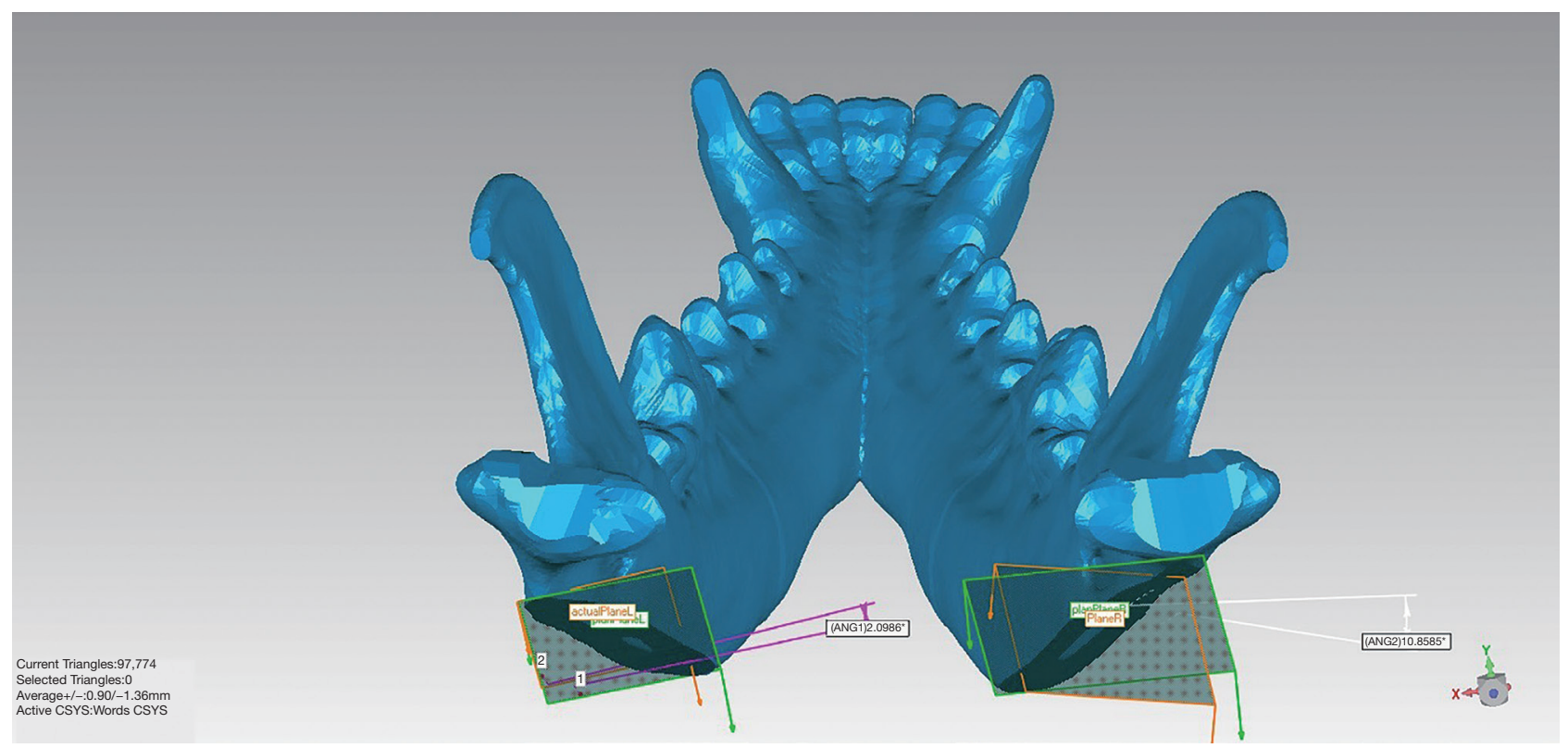

Figure 8 The osteotomy plane of postoperative CT was compared with the preoperative plan to calculate the deviation of angle.

in the traditional control group, the error of the lateral osteotomy line was $1.77 \pm 1.36 \mathrm{~mm}$, and the error of the interior osteotomy line was $1.48 \pm 1.09 \mathrm{~mm}$, angle between two planes was $8.2^{\circ} \pm 4.4^{\circ}$.

Our results revealed a great difference in accuracy along the lateral osteotomy line, with $\mathrm{P}<0.0001$, while the interior osteotomy line showed well enough result, with $\mathrm{P}<0.05$. Osteotomy angle deviation had no significant difference, with $\mathrm{P}<0.5$. The comparison of accuracy on the osteotomy line between the robot group and the manual group is shown in Figure 9. Preoperative equipment installation and preparation took 45 minutes. The robot-assisted surgery 
Table 1 Results of model experiment

\begin{tabular}{|c|c|c|c|}
\hline Model number & Position deviation (mm) & Angle deviation $\left({ }^{\circ}\right)$ & Time of installation \& navigation ( $\mathrm{min}$ ) \\
\hline Model 2 & $0.42 \pm 0.15$ & 1.2 & 20 \\
\hline Model 3 & $0.34 \pm 0.13$ & 5.0 & 10 \\
\hline Model 4 & $0.47 \pm 0.08$ & 6.4 & 15 \\
\hline Mean & $0.44 \pm 0.19$ & $3.5 \pm 2.1$ & 15 \\
\hline
\end{tabular}

Table 2 Results of animal experiment

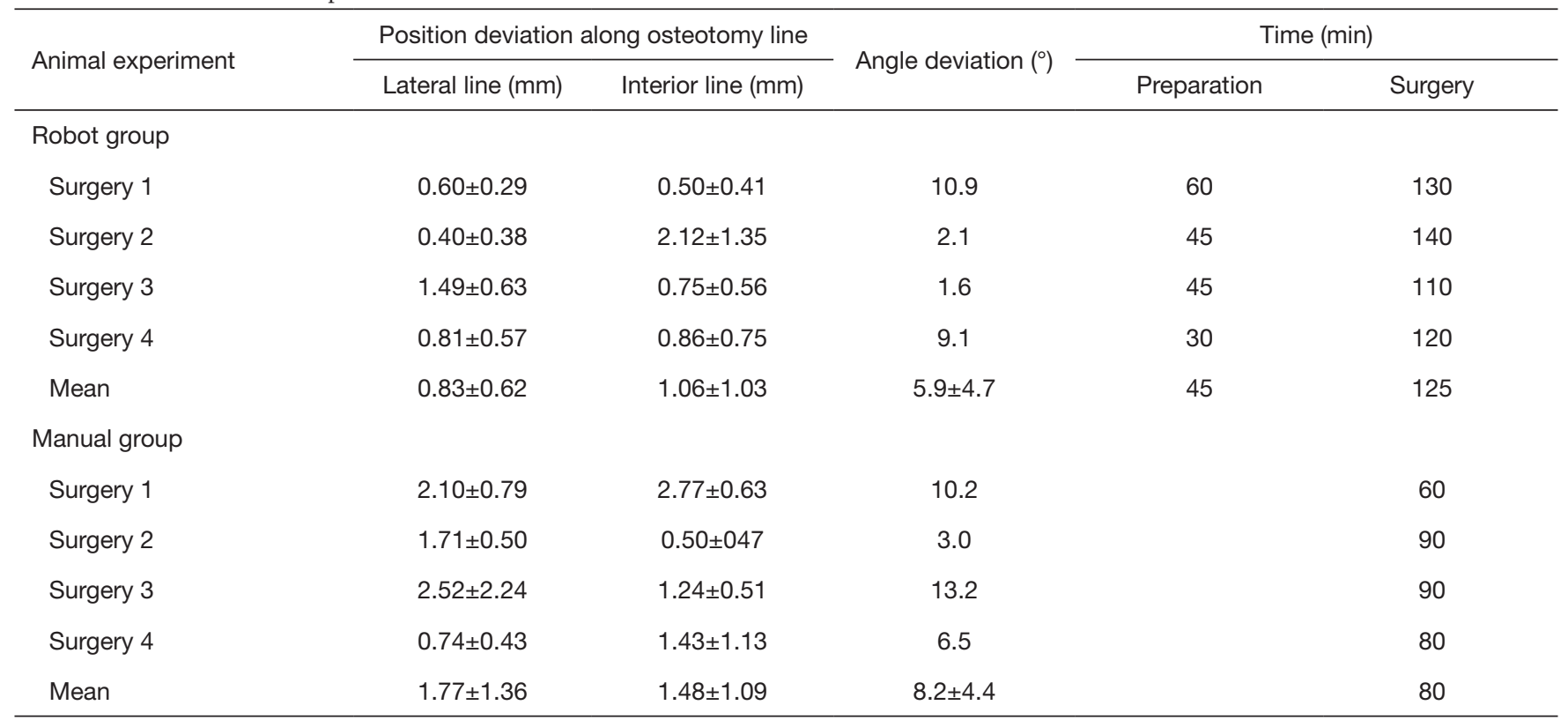

took 125 minutes on average, while the traditional approach in the control group took 80 minutes on average. Thus, the proposed approach could save 45 minutes of operation time. No postoperative complication was found in any of the experimental animals.

\section{Discussion}

Robot-assisted surgery is characterized by high accuracy and high stability; however, confined surgical areas have restrained utilization. Since the first robot-assisted surgery approved by the Federal Drug Administration (FDA) in 1994 (6), robotic and computer-guided systems have significantly improved operating practice by reducing tremors, increasing manual dexterity, and expanding the vision field. Following progressive changes, medical robotics has been identified to have high potential and the ability to bring patients real benefits due to its high accuracy positioning capability (7). With the growing demands on health systems, it is inevitable that the future of healthcare will be linked to robotics (8). Thus far, robotic systems, which have been widely adopted for urological, gynecological, rectal surgery and so on, have been considered as particularly favorable for deep and narrow spaces (9). Despite numerous technical advancements in surgical robotics over recent years, only a small proportion of surgical specialties have notably benefited from these innovations. The field of plastic and reconstructive surgery has seen little utilization of surgical robotics and computerassisted systems in the operating theatre (10). We previously 


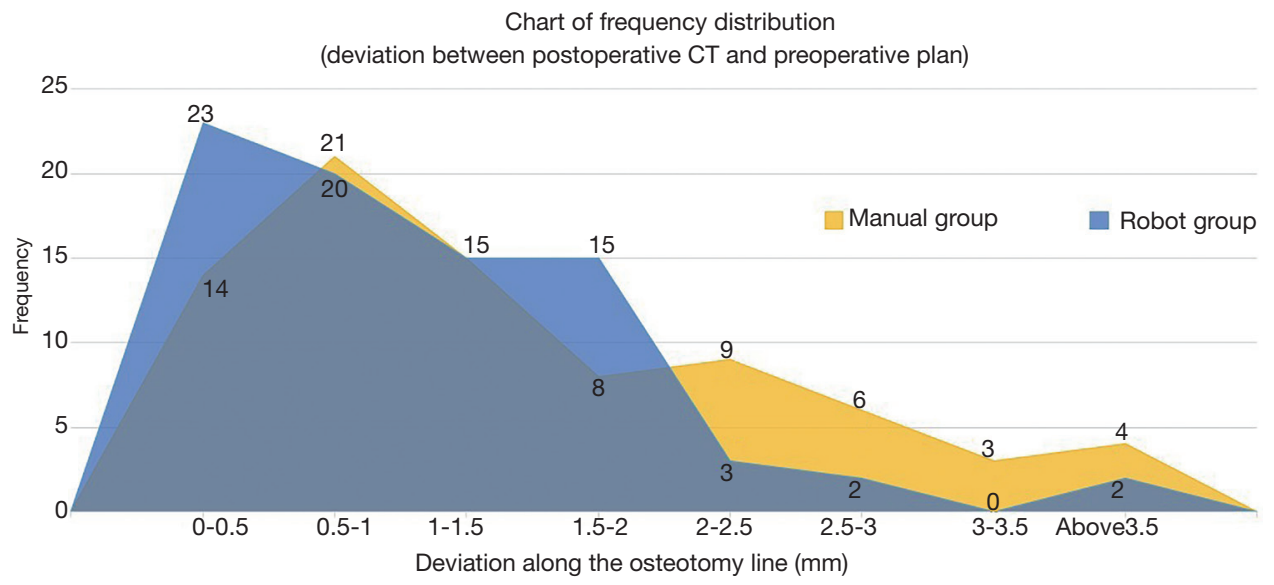

Figure 9 Compare osteotomy accuracy of the manual group and the robot group, measurement results distributed in different deviation ranges with different frequencies.

developed a robot system for automatic drilling procedures in craniofacial surgery, which achieved position error within $2 \mathrm{~mm}$ in the laboratory environment and $3 \mathrm{~mm}$ in animal experiments (11). However, before the robotassisted procedure, the registration process requires the selection of anatomical landmarks under the condition of no interference like soft tissues. Considering the subsequent clinical application, navigation technology is necessary to complete the registration quickly and accurately. In fact, as a crucial part of robot-assisted surgery, intraoperative navigation is required in most surgical robotic systems.

Most existing navigation systems are based on optical tracking techniques. An optical tracking system using infrared light is the most developed and widely practiced; however, it has some apparent limitations. Optical navigation solutions using infrared stereoscopic camera systems presuppose a clear line of (camera) sight (12), and they cannot track flexible instruments (13). In addition, the relatively large and heavy tracking tools are difficult to use in delicate surgical environments. One main barrier preventing us from adopting optical navigation for our robot system is that it's too bulky, and confined surgical areas in craniomaxillofacial surgery cannot accommodate much robotic surgical auxiliary equipment. Thus, optical navigation systems are not suitable. On the other hand, EM tracking has been successfully used in navigation completely inside the human body like in neurosurgeries and cardiovascular surgeries, such as ventricular catheter placement $(14,15)$, catheter ablation (16-18), thoracic aortic stent-graft deployment (19). This technique, which has no line-of-sight problem and is easy to handle, might supersede the widespread optical systems (12). Therefore, we combined EM navigation with robot technology for the first time and applied it to maxillofacial surgery.

To the best of our knowledge, EM systems are usually less accurate compared with optical tracking systems, and it is not as widely used in clinical practice. However, EM system accuracy has increased over the last few years, and with reductions in sensor sizes, this technology has become attractive for narrow field navigation (20). Several studies have been able to show the great potential of the EM navigation technology in craniomaxillofacial surgery. In awake craniotomy, EM navigation without head pin fixation offers the same accuracy as optical navigation (21). During orthognathic surgery, it can accurately reposition the mandibular proximal segment into its original position (22-24) or replace the maxilla after Le Fort I osteotomy without the use of intermaxillary splints $(12,25,26)$, thus reaching the accuracy of optical trackers (27). For applications within the proper working volume, EM tracking tool can help accurately transfer the preoperative plan to the intraoperative procedure.

The splint was used to perform automatic EM navigation registration through a self-developed software algorithm (28). The splint can be repeatedly and conveniently mounted and removed during the preoperative CT and the operation. The four registration steel spheres on the splint were automatically detected to align coordinate systems between the operative area and the preoperative plan. Most existing EM systems fix their tracking devices rigidly in the bone, which can result in intraoperative bleeding and tissue-damaging. The hole 
on the bone surface left by the screws may interfere with healing, while extended operation time may increase the chance of complications after the operation. By contrast, our method is non-invasive and time-saving, providing convenience for both doctors and patients.

To the concerns of many researchers, the accuracy of robot-assisted surgery using EM navigation cannot be guaranteed in non-laboratory environments due to its instability. Also, various instruments and devices in surgical scenarios are a potential source of interference. During our application, we found that interference of ferromagnetic material in the magnetic field was mostly preventable. The surgical instruments used in our operations were not specially made; just like most instruments used in other common operating rooms, they were made of 300 series stainless steel, which works well with the Aurora System according to the Aurora user guide. Navigation is only a part of the surgical process. During registration, most of the interference caused by ferromagnetic material can be avoided by temporarily removing these materials away from the surgical field. As for the unavoidable materials, such as the surgical bed frame and mandibular fixing frame, we reduced the interference as much as possible by changing the position of the magnetic field generator. Manipulation of the emitter of an EM navigation system helps to prevent interference caused by metal surgical instruments (29). The aim of our system was to solve the problem of surgeons' inability to carry out the osteotomy precisely as the preoperative plan. As a result, our robotic system successfully assisted surgeons in performing the robotassisted surgery with a deviation of less than $2 \mathrm{~mm}$ between surgical plan and post-operation CT outcome. For our mandibular angle osteotomy, such an error was completely acceptable.

Our system using EM tracking resulted feasible both in terms of precision and process flow. Preparation of this robot system was completed before the operation, including splint construction, preoperative CT, surgical planning, and robot path generation. Procedures that increased surgical time were the magnetic navigation registration and the robot positioning of the template, which took less than an hour in total. Process duration could be further reduced by increasing the proficiency of surgical personnel.

Currently, our proposed robot system was only applied to mandibular angle osteotomy, which was chosen as a representative surgery as it has a confined surgical field and requires high accuracy. Nonetheless, other operations with similar navigation range may be potential applications, while the technology of splint based automatic registration can also be used in other maxillary and mandibular surgeries. Animal experiment results were satisfactory; however, whether these outcomes can be reproduced in clinical patients still remains unclear and needs to be further explored. Without a doubt, robot-assisted surgery is a trend, while the intellectualization of medical equipment is a promising direction. Under constant development in terms of surgical simulation (30), mechatronics, and surgeonrobot interfaces, the utility of robotic assistance is likely to become preferred and even the first choice for clinical cranial maxillofacial surgery in the near future.

\section{Conclusions}

All animals survived and healed well; post-operation CT validated the system's precision, confirming more satisfying outcomes of robot-assisted mandibular angle osteotomy compared to the traditional procedure. This experiment's results show that EM navigation is feasible in the real operating environment; the procedure we developed and proposed was safe and easy on animals. The proposed procedure has the potential to be applied to clinical practice in the near future.

The main obstacle that may prevent applying this robot system in clinic is the equipment that takes up a large amount of operating room space. Also, from an economic perspective, the use of robotic systems and professionals' operation can add about $\$ 100$ to the cost of surgery.

With the development of EM navigation technology and human-robot interaction, robot-assisted surgery can be used to complete the procedures more accurately and safely, thus providing further support for high-precision surgical interventions.

\section{Acknowledgments}

Funding: This work described in this paper was supported by the project of Science and technology commission of Shanghai municipality (18DZ2201900, 19441912300/), Shanghai Municipal Education Commission-Gaofeng Clinical Medicine Grant Support (20161420) and the Clinical Research Plan of SHDC /No. SHDC2020CR3070B.

\section{Footnote}

Reporting Checklist: The authors have completed the 
ARRIVE reporting checklist. Available at http://dx.doi. org/10.21037/atm-20-6305

Data Sharing Statement: Available at http://dx.doi. org/10.21037/atm-20-6305

Conflicts of Interest: All authors have completed the ICMJE uniform disclosure form (available at http://dx.doi. org/10.21037/atm-20-6305). All authors report grants from the project of Science and technology commission of Shanghai municipality (18DZ2201900, 19441912300), grants from Shanghai Municipal Education CommissionGaofeng Clinical Medicine Grant Support [20161420], during the conduct of the study.

Ethical Statement: The authors are accountable for all aspects of the work in ensuring that questions related to the accuracy or integrity of any part of the work are appropriately investigated and resolved. Experiments were performed under a project license (No.: SH9HIEC-2017319-T239) granted by Independent Ethics Committee of Shanghai Ninth People's Hospital affiliated with the Shanghai Jiao Tong University, School of Medicine, in compliance with institutional relevant guidelines and regulations for the care and use of animals.

Open Access Statement: This is an Open Access article distributed in accordance with the Creative Commons Attribution-NonCommercial-NoDerivs 4.0 International License (CC BY-NC-ND 4.0), which permits the noncommercial replication and distribution of the article with the strict proviso that no changes or edits are made and the original work is properly cited (including links to both the formal publication through the relevant DOI and the license). See: https://creativecommons.org/licenses/by-nc-nd/4.0/.

\section{References}

1. Lei R, Wang Y, Xu S, et al. A modified intraoral and extraoral approach osteotomy for the prominent mandibular angle. J Plast Reconstr Aesthet Surg 2017;70:1091-100.

2. Yu H, Shen SG, Wang X, et al. The indication and application of computer-assisted navigation in oral and maxillofacial surgery-Shanghai's experience based on 104 cases. J Craniomaxillofac Surg 2013;41:770-4.

3. Berger M, Kallus S, Nova I, et al. Approach to intraoperative electromagnetic navigation in orthognathic surgery: A phantom skull based trial. J Craniomaxillofac Surg 2015;43:1731-6.

4. Zhu M, Liu F, Chai G, et al. A novel augmented reality system for displaying inferior alveolar nerve bundles in maxillofacial surgery. Sci Rep 2017;7:42365.

5. Balicki M, Kyne S, Toporek G, et al. Design and control of an image-guided robot for spine surgery in a hybrid OR. Int J Med Robot 2020;16:e2108.

6. Meadows M. Robots Lend a Helping Hand to Surgeons. FDA Consumer magazine 2002;36:5-10.

7. Chen CS, Hsieh MS, Chiu YW, et al. An unconstrained virtual bone clamper for a knee surgical robot using visual servoing technique. Journal of the Chinese Institute of Engineers 2010;33:379-86.

8. Mattos LS, Caldwell DG, Peretti G, et al. Microsurgery robots: addressing the needs of high-precision surgical interventions. Swiss Med Wkly 2016;146:w14375.

9. Lee SH, Lim S, Kim JH, et al. Robotic versus conventional laparoscopic surgery for rectal cancer: systematic review and meta-analysis. Ann Surg Treat Res 2015;89:190-201.

10. Tan YPA, Liverneaux P, Wong JKF. Current Limitations of Surgical Robotics in Reconstructive Plastic Microsurgery. Front Surg 2018;5:22.

11. Sun M, Chai Y, Chai G, et al. Fully Automatic RobotAssisted Surgery for Mandibular Angle Split Osteotomy. J Craniofac Surg 2020;31:336-9.

12. Berger M, Nova I, Kallus S, et al. Can electromagneticnavigated maxillary positioning replace occlusional splints in orthognathic surgery? A clinical pilot study. J Craniomaxillofac Surg 2017;45:1593-9.

13. Yaniv Z, Wilson E, Lindisch D, et al. Electromagnetic tracking in the clinical environment. Med Phys 2009;36:876-92.

14. Weiner GM, Chivukula S, Chen CJ, et al. Ommaya reservoir with ventricular catheter placement for chemotherapy with frameless and pinless electromagnetic surgical neuronavigation. Clin Neurol Neurosurg 2015;130:61-6.

15. Gilard V, Magne N, Gerardin E, et al. Comparison of electromagnetic neuronavigation system and free-hand method for ventricular catheter placement in internal shunt. Clin Neurol Neurosurg 2017;158:93-7.

16. Schwagten B, Jordaens L, Witsenburg M, et al. Initial experience with catheter ablation using remote magnetic navigation in adults with complex congenital heart disease and in small children. Pacing Clin Electrophysiol 2009;32 Suppl 1:S198-201.

17. Filgueiras-Rama D, Estrada A, Shachar J, et al. Remote 
magnetic navigation for accurate, real-time catheter positioning and ablation in cardiac electrophysiology procedures. J Vis Exp 2013;(74):3658.

18. Roudijk RW, Gujic M, Suman-Horduna I, et al. Catheter ablation in children and young adults: is there an additional benefit from remote magnetic navigation? Neth Heart J 2013;21:296-303

19. Luo Z, Cai J, Wang S, et al. Magnetic navigation for thoracic aortic stent-graft deployment using ultrasound image guidance. IEEE Trans Biomed Eng 2013;60:862-71.

20. Schicho K, Figl M, Donat M, et al. Stability of miniature electromagnetic tracking systems. Phys Med Biol 2005;50:2089-98.

21. Morsy AA, Ng WH. Awake craniotomy using electromagnetic navigation technology without rigid pin fixation. J Clin Neurosci 2015;22:1827-9.

22. Nova I, Kallus S, Berger M, et al. Computer assisted positioning of the proximal segment after sagittal split osteotomy of the mandible: Preclinical investigation of a novel electromagnetic navigation system. J Craniomaxillofac Surg 2017;45:748-54.

23. Berger M, Nova I, Kallus S, et al. Electromagnetic navigated condylar positioning after high oblique sagittal split osteotomy of the mandible: a guided method to attain pristine temporomandibular joint conditions. Oral Surg Oral Med Oral Pathol Oral Radiol 2018;125:407-14.e1.

24. Lee SJ, Yang HJ, Choi MH, et al. Real-time

Cite this article as: Sun $\mathrm{M}$, Lin $\mathrm{L}$, Chen $\mathrm{X}, \mathrm{Xu} \mathrm{C}, \mathrm{Zin} \mathrm{MA}$, Han W, Chai G. Robot-assisted mandibular angle osteotomy using electromagnetic navigation. Ann Transl Med 2021;9(7):567. doi: 10.21037/atm-20-6305 augmented model guidance for mandibular proximal segment repositioning in orthognathic surgery, using electromagnetic tracking. J Craniomaxillofac Surg 2019;47:127-37.

25. Lutz JC, Nicolau S, Agnus V, et al. A novel navigation system for maxillary positioning in orthognathic surgery: Preclinical evaluation. J Craniomaxillofac Surg 2015;43:1723-30.

26. Berger M, Nova I, Kallus S, et al. Electromagnetic navigated positioning of the maxilla after Le Fort I osteotomy in preclinical orthognathic surgery cases. Oral Surg Oral Med Oral Pathol Oral Radiol 2017;123:298-304.

27. Seeberger R, Kane G, Hoffmann J, et al. Accuracy assessment for navigated maxillo-facial surgery using an electromagnetic tracking device. J Craniomaxillofac Surg 2012;40:156-61.

28. Lin L, Gao Y, Chai G, et al. Electromagnetic Navigation in Craniofacial Surgery Based on Automatic Registration of Dental Splints. J Craniofac Surg 2020;31:393-6.

29. Takenaka T, Toyota S, Kuroda H, et al. Freehand Technique of an Electromagnetic Navigation System Emitter to Avoid Interference Caused by Metal Neurosurgical Instruments. World Neurosurg 2018;118:143-7.

30. Lin L, Sun M, Gao Y, et al. Modeling and Clinical Application of Incision Space in Facial Contour Surgery. J Craniofac Surg 2020;31:377-80. 